\title{
Oropharyngeal, Hypopharyngeal and Cervical Esophageal Reconstruction: An Experience of Pedicle Flaps
}

\author{
Farrukh Aslam Khalid, Muhammad Saleem, Muhammad Amin Yousaf, \\ Abdul Malik Mujahid, Imran Shahzad and Moazzam Nazeer Tarar
}

\begin{abstract}
Objective: To review the experience of single-stage reconstruction following pharygolaryngectomy and cervical esophageal defect with pedicle flaps in a tertiary care centre.

Study Design: Retrospective study.

Place and Duration of Study: Jinnah Burn and Reconstructive Surgery Centre, Lahore, from June 2007 to June 2017. Methodology: All patients who underwent oropharyngeal, hypopharyngeal and cervical esophageal reconstruction with pedicled flaps were included.

Results: Thirty-two reconstructions were done, of which $16(50.0 \%)$ were supraclavicular flaps, 12 (37.5\%) were pectoralis major myocutaneous flaps (PMMF) and 4 (12.5\%) platysma myocutaneous flaps (PMF). Among these, 24 (75\%) were males and $8(25 \%)$ females. The mean hospital stay was $18.75 \pm 5.45$ days. Complications were noted in $12(37.5 \%)$, salivary fistula being the most frequent found in 6 (18.75\%). Wound dehiscence was noted in 4 (12.5\%), partial flap necrosis and wound infection present in $1(3.1 \%)$ each.

Conclusion: Oropharyngeal, hypopharyngeal and cervical esophageal reconstruction with pedicle flaps is still very useful and safe to perform, associated with minimal flap and donor site complications.
\end{abstract}

Key Words: Pharyngeal reconstruction, Pedicled flap, Supraclavicular flap, Pectoralis myocutaneous flap.

\section{INTRODUCTION}

Oropharyngeal, hypopharyngeal and cervical esophageal defects after cancer surgery, trauma and stricture are a constant challenge for reconstruction. This group of patients is difficult due to the disease associated morbidity and the functional and aesthetic defects. ${ }^{1}$ Numerous flaps have been described including myocutaneous (pectoralis major, trapezius and latissimus dorsi), fasciocutaneous (deltopectoral, supraclavicular artery) and microvascular (radial forearm, anterolateral thigh, parascapular, abdominal, jejunal) flaps have been described. The selection of flaps for reconstruction depends on the type and location of the defect, patient characteristics and the experience of the surgical team.1-12 Pharyngeal defects are mostly due to cancer surgery, trauma and benign diseases like corrosive stricture. 1 The fundamental goals of any reconstruction of a pharyngeal defect are to restore the integrity of digestive track for swallowing; to maintain phonation, either by conservation of larynx or by any rehabilitation technique in case of total laryngectomy; and to perform the

Department of Plastic Surgery, Jinnah Burn and Reconstructive Surgery Center / Allama Iqbal Medical College, Lahore,

Pakistan

Correspondence: Dr. Farrukh Aslam Khalid, Department of

Plastic Surgery, Jinnah Burn and Reconstructive Surgery

Center, Lahore, Pakistan

E-mail: drfarrukhaslam@gmail.com

Received: February 16, 2018; Accepted: September 9, 2018 technique in a single surgical operation with minimum morbidity and mortality. 2,5

Trotter in 1913 was the first to use cervical skin flaps for surgical reconstruction of pharynx, ${ }^{4}$ and now there is the era of free vascularized flaps. ${ }^{7}$ Regional flaps are still a valid option for this reconstruction. In this study, the use of pedicled flaps for pharyngeal and cervical esophageal reconstruction in 32 patients is reported.

Although in this era of microvascular surgery, the free tissue transfer is the standard of care, but needs long operative time, potential risk of complete flap loss, difficulty of monitoring of buried flaps, need of major resources, difficulty to accommodate in busy surgery lists and, in case of previous radiation, difficulty to dissect out the appropriate donor vessels. ${ }^{13-15}$

The need of local flap remains as it is adjacent to the area of reconstruction, with minimal operative time, need of limited resources, and no need of extensive monitoring. ${ }^{16-18}$

The aim of this study was to review the outcome of reconstructive procedures for flap survival and the complications.

\section{METHODOLOGY}

The data of patients undergoing oropharyngeal, hypopharyngeal and cervical esophageal reconstruction was retrospectively analysed from June 2007 to June 2017 after approval of Hospital Ethical Committee. The medical records were reviewed and data were collected for the cause of pharyngo-esophageal defect, patient 
characteristics, location and size of the defect, the reconstruction method, complications of reconstruction and hospital stay. All patients with pharyngo-esophageal reconstruction, using pedicle flaps, were included in the study. The mean follow-up period was 24 months with a minimum of 6 months.

Data was entered and analysed in SPSS version 21.0. Mean + standard deviation (SD) was calculated for continuous variables like age and duration of hospital stay. Frequencies and percentages were calculated for categorical variables like gender, type of flaps used, and complications. Chi-square test was used to assess the significance for complications among type of flaps with $p<0.05$, which was statistically considered as significant.

\section{RESULTS}

A total of 32 patients were included, with a mean age of $42.25 \pm 13.05$ years (ranging from 16-70 years), of which $24(75 \%)$ were males and $8(25 \%)$ females. Supraclavicular flap was used in $16(50 \%)$ cases, pectoralis major myocutaneous flap in $12(37.5 \%)$ and platysma myocutaneous flap in $4(12.0 \%)$ cases. Fourteen $(43.7 \%)$ patients were smokers, and $9(28.1 \%)$ were diabetics, whereas $10(31.2 \%)$ patients had previous radiation therapy. Mean operation time was 142 minutes (ranging from 90 to 210 minutes). The maximum circumferential defect reconstructed was $10 \mathrm{~cm}$. The demographic and clinical characteristics of patients are given in Table I.

All patients presented to us after primary surgery, 16 $(50.0 \%)$ were operated for malignancy, 12 (37.5\%) for corrosive stricture, and 04 (12.5\%) for trauma.

All patients were followed up for long-term complications like stenosis, ranging from 6 months to 5 years with the mean follow-up of 2 years. The mean hospital stay was $18.75 \pm 5.45$ days (ranging from $15-45$ days). Patients with complications stayed longer than the patients with uneventful recovery. Representative case pictures are shown in Figures 1 and 2.

Complications were noted in $12(37.5 \%)$ patients, the most common being salivary fistula, in $6(18.6 \%)$ cases. Partial flap necrosis occurred in $1(3.2 \%)$ case, i.e. supra-

Table I: Etiology, location, size and type of defect and reconstruction with complications of flap.

\begin{tabular}{|c|c|c|c|c|c|c|c|}
\hline Gender & Age & Etiology & Location of defect & Type of defect & $\begin{array}{l}\text { Size of } \\
\text { defect }\end{array}$ & Reconstruction & Complication \\
\hline Male & 48 & Tumor & Hypopharynx and cervical esophagus & Anterior and lateral walls & $7 \mathrm{~cm}$ & Pectoralis major myocutaneous & \\
\hline Male & 29 & Corrosive & Hypopharynx & Circumferential & $8 \mathrm{~cm}$ & Pectoralis major myocutaneous & \\
\hline Male & 52 & Tumor & Hypopharynx and cervical esophagus & Circumferential & $7.5 \mathrm{~cm}$ & Pectoralis major myocutaneous & Dehiscence \\
\hline Female & 40 & Tumor & Hypopharynx and cervical esophagus & Anterior and lateral walls & $6 \mathrm{~cm}$ & Supraclavicular flap & \\
\hline Male & 38 & Trauma & Hypopharynx & Anterior and right lateral wall & $5 \mathrm{~cm}$ & Pectoralis major myocutaneous & Fistula \\
\hline Male & 54 & Tumor & $\begin{array}{l}\text { Oropharynx, hypopharynx and } \\
\text { cervical esophagus }\end{array}$ & Circumferential & $10 \mathrm{~cm}$ & Supraclavicular flap & \\
\hline Male & 52 & Tumor & Hypopharynx and cervical esophagus & Circumferential & $8 \mathrm{~cm}$ & Pectoralis major myocutaneous & \\
\hline Female & 47 & Tumor & Hypopharynx and cervical esophagus & Circumferential & $6.5 \mathrm{~cm}$ & Supraclavicular flap & Partial Necrosis \\
\hline Female & 29 & Corrosive & Hypopharynx & Circumferential & $3 \mathrm{~cm}$ & Platysma myocutaneous flap & \\
\hline Male & 30 & Corrosive & Hypopharynx & Circumferential & $4 \mathrm{~cm}$ & Pectoralis major myocutaneous & \\
\hline Male & 29 & Trauma & Hypopharynx & Anterior wall & $5 \mathrm{~cm}$ & Supraclavicular flap & \\
\hline Male & 60 & Tumor & Hypopharynx and cervical esophagus & Circumferential & $10 \mathrm{~cm}$ & Supraclavicular flap & Fistula \\
\hline Male & 41 & Corrosive & Hypopharynx & Anterior and lateral walls & $8 \mathrm{~cm}$ & Supraclavicular flap & \\
\hline Female & 32 & Corrosive & Hypopharynx & Circumferential & $3.5 \mathrm{~cm}$ & Platysma myocutaneous flap & Infection \\
\hline Male & 70 & Tumor & Hypopharynx and cervical esophagus & Circumferential & $7 \mathrm{~cm}$ & Pectoralis major myocutaneous & \\
\hline Male & 44 & Trauma & Hypopharynx & Anterior and lateral walls & $3.5 \mathrm{~cm}$ & Platysma myocutaneous flap & Dehiscence \\
\hline Male & 62 & Tumor & Hypopharynx and cervical esophagus & Circumferential & $6.5 \mathrm{~cm}$ & Supraclavicular flap & \\
\hline Female & 16 & Corrosive & Hypopharynx & Anterior and lateral walls & $4 \mathrm{~cm}$ & Supraclavicular flap & \\
\hline Male & 46 & Tumor & Hypopharynx and cervical esophagus & Circumferential & $5.5 \mathrm{~cm}$ & Supraclavicular flap & \\
\hline Female & 27 & Corrosive & Hypopharynx & Anterior and lateral walls & $5 \mathrm{~cm}$ & Supraclavicular flap & Fistula \\
\hline Male & 48 & Tumor & Hypopharynx and cervical esophagus & Circumferential & $7 \mathrm{~cm}$ & Pectoralis major myocutaneous & \\
\hline Male & 40 & Corrosive & Hypopharynx & Anterior and lateral walls & $4 \mathrm{~cm}$ & Supraclavicular flap & \\
\hline Male & 49 & Tumor & Hypopharynx and cervical esophagus & Circumferential & $7 \mathrm{~cm}$ & Pectoralis major myocutaneous & Dehiscence \\
\hline Female & 26 & Corrosive & Hypopharynx & Anterior and lateral walls & $4 \mathrm{~cm}$ & Supraclavicular flap & \\
\hline Male & 29 & Corrosive & Hypopharynx and cervical esophagus & Circumferential & $5 \mathrm{~cm}$ & Supraclavicular flap & Fistula \\
\hline Male & 36 & Corrosive & Hypopharynx & Anterior and lateral walls & $5 \mathrm{~cm}$ & Supraclavicular flap & \\
\hline Male & 57 & Tumor & Hypopharynx and cervical esophagus & Circumferential & $8 \mathrm{~cm}$ & Pectoralis major myocutaneous & Fistula \\
\hline Male & 35 & Trauma & Hypopharynx and cervical esophagus & Anterior wall & $5 \mathrm{~cm}$ & Supraclavicular flap & \\
\hline Male & 53 & Tumor & Hypopharynx and cervical esophagus & Circumferential & $8 \mathrm{~cm}$ & Pectoralis major myocutaneous & $\begin{array}{l}\text { Dehiscence+ } \\
\text { Fistula }\end{array}$ \\
\hline Female & 23 & Corrosive & Hypopharynx & Circumferential & $3 \mathrm{~cm}$ & Platysma myocutaneous flap & \\
\hline Male & 51 & Tumor & Hypopharynx and cervical esophagus & Circumferential & $7 \mathrm{~cm}$ & Pectoralis major myocutaneous & \\
\hline Male & 59 & Tumor & Hypopharynx and cervical esophagus & Circumferential & $9 \mathrm{~cm}$ & Supraclavicular flap & \\
\hline
\end{tabular}




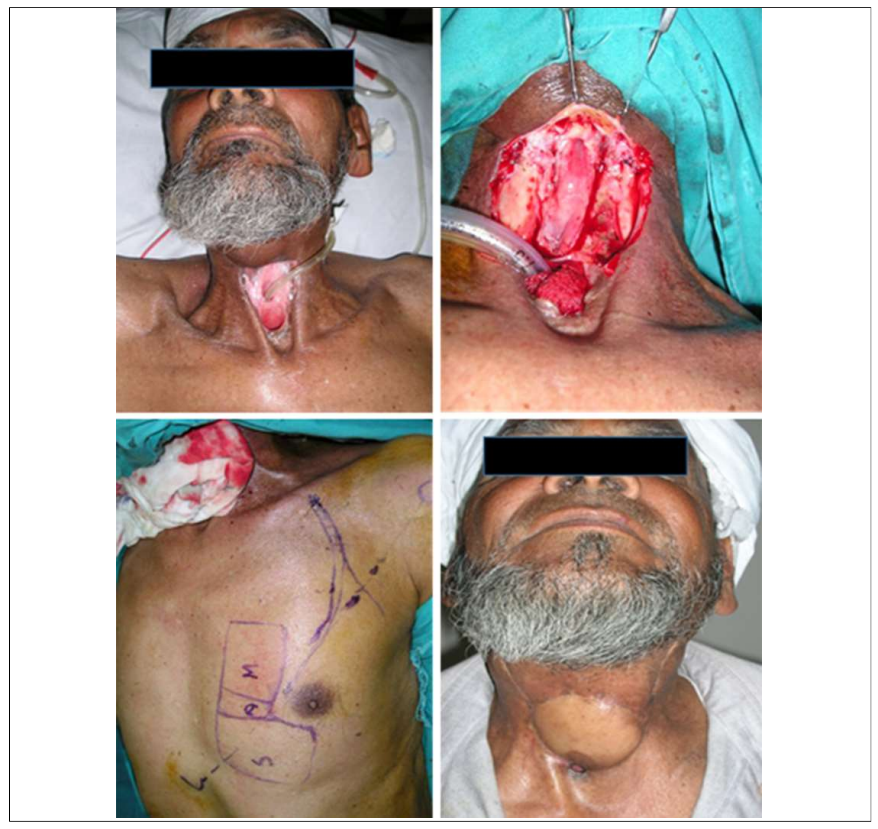

Figure 1: Seventy-year male presented with cervical esophageal fistula after laryngectomy and hypopharyngeactomy. Defect was covered with double paddle myocutaneous pectoralis major flap.
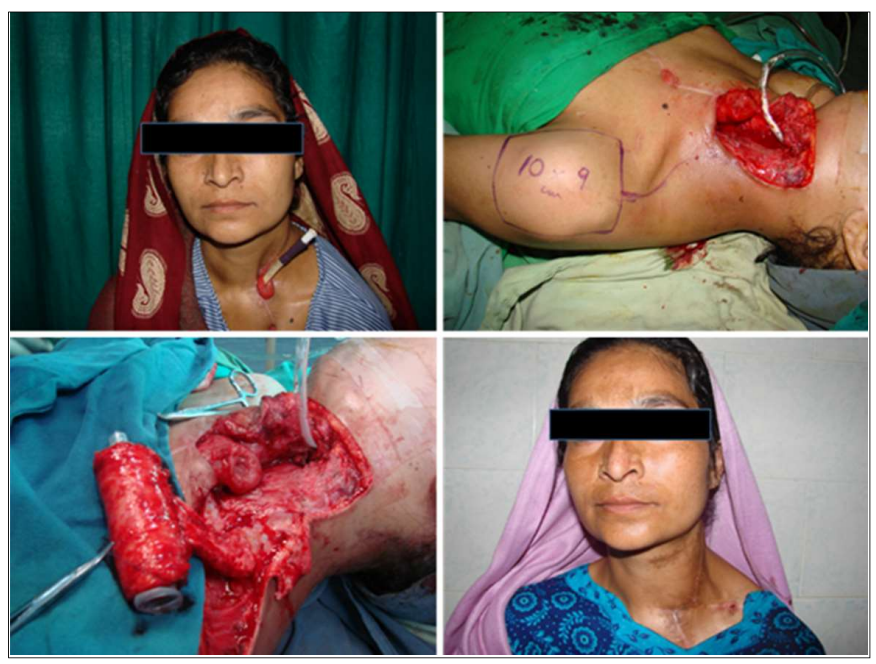

Figure 2: Twenty-five-year female presented with jejunostomy loupe into the neck after esophgectomy, but could not join it with the cervical part of esophagous. So supraclavicular flap was tubed to act as a bridge.

clavicular artery flaps. After flap debridement, the defect was covered with myocutaneous pectoralis major flap. Dehiscence occurred in $4(25.0 \%)$ cases. Stenosis was not observed in any case. The type of complications is shown in Table I.

\section{DISCUSSION}

Pharyngeal and cervical esophageal disorders lead to great morbidity which makes the reconstruction a challenge for the reconstructive surgeons. Debate is still going on for the most appropriate tissue for reconstruction to establish continuity between the oral cavity and the esophagus. Most pharyngeal defects present after tumor excision. The ideal reconstruction in these cases is usually one-stage procedure with reliable and robust tissue for early functional recovery and ability to tolerate postoperative radiotherapy. The availability of tissue for reconstruction ranges from primary closure to free tissue transfers, but depends on the extent of defect, neck dissection, previous radiation and patient's general health.

Contemporary options for pharyngoesophageal reconstruction are pedicled flaps and free flaps (enteric and fasciocutaneous). 1 The most common fasciocutaneous free flaps for pharyngoesophageal reconstruction are the free radial forearm and the anterolateral thigh flap. Traditionally, for circumferential defects of hypopharynx and esophagus above the thoracic inlet, enteric flaps, i.e. free jejunal transfer have been used. For defects below the thoracic inlet, reconstruction has been done using gastric pull-ups. The free enteric flaps have been the treatment of choice over the fasciocutaneous flaps but the added morbidity of a laparotomy and intestinal anastomosis decreases their use. The additional advantages of fasciocutaneous flaps are less donor site morbidity, better trachea-esophageal speech quality, and comparable stricture (12-27\%) and fistula rates (0-13\%). 1,19 All these flaps need long operative time, microsurgery facility and invasive monitoring for flaps and are prone to complete flap failure. 13

This study suggests that regional flaps are still valid options for hypopharyngeal and cervical esophageal reconstruction associated with low morbidity, and offering comparable results with the free flaps. In comparison to free flaps, the regional flaps are easy to harvest, technically less demanding and are desirable in patients with advanced age, extensive disease, comorbidities or poor nutritional status. Furthermore, the radial forearm flap has significant donor site morbidity, and the anterolateral thigh flap skin paddle is usually thick especially in females and difficult to tube. ${ }^{20}$

For the reconstruction of the circumferential pharyngolaryngectomy defects, double paddle myocutaneous pectoralis major flaps and tube was made by joining the two paddles, were used. The folding of this flap for the reconstruction of circumferential defect is difficult and resulted in more complications than for reconstruction of non-circumferential defects. In females, the breast tissue results in less reliable skin paddle and the resulting loss of contour of breast and scar are unacceptable for female patients. In one case, jejunal pull-up was done to reconstruct after corrosive stricture of pharynx and cervical esophagous, but the cervical part got necrozed; later, the defect was reconstructed with tubed supraclavicular flap. Four cases presented with post-laryngectomy/post-radiotherapy pharyngeal fistulas as a manifestation of tumour recurrence. Wide excision and reconstruction was done with pedicle flaps. The most important observation in our study was that the complication rate was not too high, despite performing 
the procedures in severely disease-compromised patients. The percentage of satisfactory results in regional flaps was $95 \%$.

Mamoon et al. reported $21 \%$ fistula rate with no flap loss in his series of 14 reconstructions with pectoralis major myocutaneous flap. ${ }^{16}$ Lakhera et al. recommended the use of patch pharyngoplasty by pectoralis major flap for the defect up to $3.5 \mathrm{~cm} .{ }^{21} \mathrm{In}$ their series, the fistula rate was $27.1 \%$. Murray et al. reported in a study the overall fistula rate of about $13 \%$ in all fasciocutaneous flaps, with $16.4 \%$ in the anterolateral thigh flap and $14.4 \%$ in radial forearm flap patients. ${ }^{3} \mathrm{Yu}$ and Robb reported a fistula rate of $33 \%$; and after their technique modification; fistula rate decreased to $8 \% .{ }^{11} \mathrm{In}$ the other large series, the overall fistula rate ranged from 8 to $22 \%$ for free jejunal flaps.9,11,20,22 Richmon et al. reported the fistula rate for pectoralis major flaps range from $0-47 \% .23$ In this study, fistula rate was $18.6 \%$. According to a study by Chang et al., $1265 \%$ of all fistulas closed spontaneously, which is consistent with the rate cited in other reports. ${ }^{23,24}$ In this study, all fistulas were managed conservatively.

Szudek et al. noted $37 \%$ complication rate in platysma myocutaneous flap with increased rate of infection when used for the pharyngeal reconstruction. ${ }^{25}$

Liu et al. suggested supraclavicular flap as ideal flap for pharyngeal reconstruction for being thin, easy to harvest, fold on itself, and good for circumferential defects with minimal donor site morbidity. ${ }^{15}$

The stricture formation after pectoralis major flap reported by Mamoon et al. was 29\%.16 While in fasciocutaneous flaps, it varies considerably between $5-40 \% .26-28$ Murray et al. reported the overall stricture rate of $16.3 \%$ in all fasciocutaneous flaps and $11.9 \%$ rate in anterolateral thigh flaps. ${ }^{3}$ While in jejunal flap, it has been reported ranging from $15-22 \% .20,22 \mathrm{Yu}$ and Robb reported $12 \%$ fistula rate in circumferential defects reconstructed with anterolateral thigh flap, ${ }^{11}$ but in this study none of the patients developed stricture.

These series suggest that despite minimal complication quoted with free microsurgical reconstruction, yet the pedicled flaps are useful alternative in selected cases, and are quick to perform with lesser secondary procedure requirement. In this study, the pectoralis major flap is better in male patients with noncircumferential reconstruction with previous neck dissection or radiation to neck. Similarly, the platysma myocutaneous flap is better for small defects with no previous trauma or radiation to the donor area. The supraclavicular flap is better for circumferential defects in either gender.

\section{CONCLUSION}

In the light of the above considerations, pedicled flaps have comparable results with free fasciocutaneous flaps with overall high success rate. The pedicled flap is onestage operation with quick flap raising, reliability; and is ideal for patients with significant medical history and old age. The pedicled flap with low complication rate and less donor site morbidity makes these flaps, in authors' opinion, a valid option for reconstruction of oropharyngeal, hypopharyngeal and cervical esophageal defects.

\section{REFERENCES}

1. Bakamjian VY. A two-stage method for pharyngoesophageal reconstruction with a primary pectoral skin flap. Plast Reconstr Surg 1965; 36:173-84.

2. Ong GB, Lee TC. Pharyngogastric anastomosis after oesophagopharyngectomy for carcinoma of the hypopharynx and cervical oesophagus. Br J Surg 1960; 48:193-200.

3. Murray D, Novak C, Neligan P. Fasciocutaneous free flaps in pharyngolaryngo-oesophageal reconstruction: A critical review of literature. J Plast Reconstr Aesthet Surg 2008; 61:1148-56.

4. Harii K, Ebihara S, Ono I, Saito H, Terui S, Takato T. Pharyngoesophageal reconstruction using a fabricated forearm-free flap. Plast Reconstr Surg 1985; 75:463-76.

5. Koshima I, Fukuda H, Yamamato H, Moriguchi T, Soeda S, Ohta S. Free anterolateral thigh flaps for reconstruction of head and neck defects. Plast Reconstr Surg 1993; 92:421-8.

6. Li KK, Salibian AH, Allison G, Krugman MK, Armstrong W, Wong $\mathrm{B}$, et al. Pharyngoesophageal reconstruction with the ulnar forearm flap. Arch Otolaryngol Head Neck Surg 1998; 124:1146-51.

7. Endo T, Nakayama Y. Pharyngoesophageal reconstruction: A clinical comparison between free tensor fasciae latae and radial forearm flaps. J Reconstr Microsurg 1997; 13:93-7.

8. Schipper J, Ridder GJ, Boedeker C, Fradis M, Golz A, Gellrich NC. Lateral upper arm free flap for primary reconstruction of pharyngeal defects in ablative oncological surgery: Report of six consecutive cases. Ann Otol Rhinol Laryngol 2003; 112:611-6.

9. Disa JJ, Pusic AL, Mehrara BJ. Reconstruction of the hypopharynx with the free jejunum transfer. $J$ Surg Oncol 2006; 94:466-70

10. Babar-Craig H, Clarke PM, Wood SH. Pharyngolaryngectomy using a free jejunal graft: A new anastomosis technique. Clin Otolaryngol 2008; 33:130-3.

11. Yu P, Robb GL. Pharyngoesophageal reconstruction with the anterolateral thigh flap: A clinical and functional outcomes study. Plast Reconstr Surg 2005; 116:1845-55.

12. Chang DW, Hussussian C, Lewin JS, Youssef AA, Robb GL, Reece GP. Analysis of pharyngocutaneous fistula following free jejunal transfer for total laryngopharyngectomy. Plast Reconstr Surg 2002; 109:1522-7.

13. Saussez S, Cuno A, Urbain F, Chantrain G, Lequeux T. Reconstruction of circumferential oro- and hypo-pharyngeal defects with U-shaped pectoralis major myocutaneous flap. Otolaryngol Head Neck Surg 2006; 134:823-9.

14. Jegoux F, Ferron C, Malard O, Espitalier F, Beauvillain de montreuil $C$. Reconstruction of circumferential pharyngolaryngectomy using a 'horseshoe-shaped' pectoralis major myocutaneous flap. $J$ Laryngol Otol 2007; 121:483-8. 
15. Liu PH, Chiu ES. Supraclavicular artery flap a new option for pharyngeal reconstruction. Ann Plast Surg 2009; 62:497-501.

16. Rashid M, Hanif MS, Rashid D, Ahmad B, Illahi I, Aslam R. Reconstruction of hypopharyngeal defects with pectoralis major myocutaneous Flap. J Coll Physicians Surg Pak 2002; 12:425-8.

17. Amin MM, Naseer U, Akhtar A, Awan AA. Pectoralis major myocutaneous flap for reconstruction of major neck defects. J Surg Pak (Int) 2014; 19:70-4.

18. Khan F, Hameed $H$, Shah SA, Khan N. Head and neck reconstruction: Our experience of pectoralis major myocutaneous pedicled flap. Professional Med J 2011; 18:310-6.

19. Rizvi TA, Rashid M, Ahmed B, Haq EU, Sarwar SU, Zia-ulIslam MZ, et al. Quality of life assessment in patients with locally advanced head and neck malignancy after ablative surgery and reconstruction with microvascular-free faps. J Coll Physicians Surg Pak 2009; 19:108-12.

20. Reece GP, Schusterman MA, Miller MJ, Kroll SS, Robb GL, Baldwin BJ, et al. Morbidity and functional outcome of free jejunal transfer reconstruction for circumferential defects of the pharynx and cervical esophagus. Plast Reconstr Surg 1995; 96:1307-16.

21. Lakhera KK, Shenoy AM, Chavan P, Siddappa K. PMMC patch pharyngoplasty reconstruction after laryngectomy: Our experience at a regional cancer institute. Indian J Otolaryngol Head Neck Surg 2015; 67:170-2.

22. Coleman JJ III, Searles JM Jr, Hester TR, Nahal F, Zubowicz V, McConnel FMS, et al. Ten years experience with free jejunal autograft. Am J Surg 1987; 154:394-8.

23. Richmon JD, Brumund KT. Reconstruction of the hypopharynx: current trends. Curr Opin Otolaryngol Head Neck Surg 2007; 15:208-12.

24. Schusterman MA, Shestak K, deVries EJ. Reconstruction of the cervical esophagus: Free jejunal transfer versus gastric pull-up. Plast Reconstr Surg 1990; 85:16-21.

25. Szudek J, Taylor SM. Systematic review of the platysma myocutaneous flap for head and neck reconstruction. Arch Otolaryngol Head Neck Surg 2007; 133:655-61.

26. Anthony JP, Singer MI, Mathes SJ. Pharyngoesophageal reconstruction using the tubed free radial forearm flap. Clin Plast Surg 1994; 21:137-47.

27. Scharpf J, Esclamado RM. Reconstruction with radial forearm flaps after ablative surgery for hypopharyngeal cancer. Head Neck 2002; 25:261-6.

28. Cho BC, Kim M, Lee JH, Byun JS, Park JS, Baik BS. Pharyngoesophageal reconstruction with a tubed free radial forearm flap. J Reconstr Microsurg 1998; 14:535-40. 